\title{
Indicadores de sustentabilidade de usinas térmicas na Amazônia: estudo de caso em Rondônia*
}

\author{
Cristiano Torres do Amaral" \\ Artur de Souza Moret** \\ José Manuel Carvalho Marta****
}

Recebido: 24/01/2018 Aceito: 25/06/2019

https://doi.org/10.22395/rium.v19n36a10

\begin{abstract}
Resumo
Este artigo apresenta uma metodologia de análise de sustentabilidade de usinas térmicas, com destaque para o sistema isolado na Amazônia, no estado de Rondônia, Brasil. O trabalho descreve indicadores para a construção de um indicador geral de sustentabilidade que possibilita classificar as microgrids térmicas de acordo com a contribuição ambiental, social e econômica. Neste estudo, foram utilizados dados oficiais e levantamento de dados junto às concessionárias de distribuição de energia elétrica. A modelagem proposta contribui para definir políticas para o controle de gases efeito estufa, a mitigação de riscos, o desenvolvimento de novas tecnologias e a distribuição de recursos públicos para investimentos em fontes alternativas de energia. O projeto de pesquisa foi desenvolvido pelo Grupo de Pesquisa Energia Sustentável Renovável da Universidade Federal de Rondônia.
\end{abstract}

Palavras-chave: desenvolvimento sustentável; indicadores; termoelétricas.

\footnotetext{
* Artigo de pesquisa Aplicação de Ferramenta de Análise de Sustentabilidade de Sistemas Microgrids na Amazônia (tese de doutorado). Fonte dos recursos: Universidade Federal de Rondônia, Centro Gestor e Operacional do Sistema de Proteção da Amazônia, Fulbright Scholar Program, Coordenação de Aperfeiçoamento de Pessoal de Nível Superior. Pesquisa realizada entre março de 2016 e novembro de 2018 pelo Grupo de Pesquisa Energia Renovável Sustentável (ESRPG).

** Doutor em Desenvolvimento Regional e Meio Ambiente. Chefe do Setor Técnico, Centro Gestor e Operacional do Sistema de Proteção da Amazônia, Porto Velho-RO, Brasil. E-mail: cristiano.amaral@sipam.gov.br. Orcid https://orcid.org/0000-0002-1335-7063

*** Doutor em Planejamento de Sistemas Energéticos. Professor titular, Universidade Federal de Rondônia, Porto Velho-RO, Brasil. E-mail: amoret@unir.br. Orcid https://orcid.org/0000-0001-7371-5486

**** Doutor Planejamento de Sistemas Energéticos. Professor associado, Universidade Federal de Rondônia, Porto Velho-RO, Brasil. E-mail: jmanuelmarta@gmail.com. Orcid https://orcid.org/0000-0002-8160-7722
} 


\title{
Indicadores de sostenibilidad de las termoeléctricas en la Amazonía: estudio de caso en Rondônia
}

\begin{abstract}
Resumen
El artículo presenta una metodología de análisis de sostenibilidad de plantas térmicas, con destaque para el sistema aislado en la Amazonía, en el estado de Rondônia, Brasil. El documento describe los indicadores para la construcción de un indicador general de sostenibilidad que permita clasificar las microrredes com termoeléctricas de acuerdo con la contribución ambiental, social y económica. En este estudio utilizamos datos oficiales y recopilación de datos con las empresas de distribución de electricidad. El modelo propuesto contribuye a la definición de políticas para el control de gases de efecto invernadero, la mitigación de riesgos, el desarrollo de nuevas tecnologías y la distribución de recursos públicos para inversiones en fuentes alternativas de energía. El proyecto de investigación fue desarrollado por el Grupo de Investigación de Energía Renovable Sostenible de la Universidade Federal de Rondônia.
\end{abstract}

Palabras clave: desarrollo sostenible; indicadores; termoeléctrico.

\section{Sustainability Indicators of the Amazonian Thermoelectrics: the Rondônia's Case Study}

\begin{abstract}
This article presents a methodology for the analysis of the sustainability of thermoelectric plants with a highlight on the isolated system in the Amazon, in the state of Rondônia, Brasil. The document describes the indicators for the constructions of a general indicator of sustainability that allows the classification of micro-networks with thermoelectrics according to their environmental, social and, economic contributions. In this study we use official data and polls made to the electric distribution companies. The model proposed in this paper contributes to the definition of policies for the control of greenhouse gases, mitigation of risks, new technologies development and, the distribution of public resources for alternative energy sources research. This research project was developed by the Research Group on Renewable and Sustainable Energy of the Universidade Federal de Rondônia.
\end{abstract}

Keywords: sustainable development; indicators; thermoelectric. 


\section{INTRODUÇÃO}

Indicadores de sustentabilidade têm por objetivo mensurar a contribuição de um processo ou sistema para o desenvolvimento ambiental, social e econômico em uma comunidade, localidade ou região. Existem modelos com indicadores e variáveis que avaliam sistemas de produção industriais, agrícolas e geração de energia em geral. Contudo, esses modelos não contam com variáveis que permitem avaliar a sustentabilidade de usinas térmicas que atendem áreas isoladas na Amazônia [1]. Atualmente, existem diferentes sistemas microgrids na Amazônia, em especial, no estado de Rondônia, Brasil. A modelagem da sustentabilidade dos sistemas isolados de geração de energia na Amazônia contribui para a definição de políticas de controle de emissão de gases efeito estufa (GEE), a migração de tecnologia e a alocação de recursos públicos para priorizar investimentos em fontes alternativas de energia.

No modelo proposto neste texto, a análise de sustentabilidade é realizada a partir da avaliação de variáveis ambiental, social e econômica. Essas variáveis são responsáveis pela construção de indicadores de sustentabilidade para cada um desses temas, bem como pela criação de um índice geral de sustentabilidade que classifica os sistemas em três níveis sustentabilidade.

A sustentabilidade das microgrids é avaliada a partir de uma matriz de indicadores ambientais, sociais e econômicos. Os dados utilizados para determinar as variáveis dos indicadores foram obtidos por meio de pesquisa nas bases oficiais, isto é, dados primários e secundários fornecidos entre os principais atores envolvidos: unidades gestoras de microgrids, concessionária de energia, prestadores de serviço e unidades consumidoras.

Este trabalho inova com a apresentação de variáveis que têm aplicação na Amazônia, descrevendo, por exemplo, a contribuição para um sistema isolado de geração de energia na erradicação de doenças locais ou para a geração de empregos nas áreas de floresta. Essa contribuição pode ser expressa, por exemplo, na relação entre cada variável e o número de ligações de energia de um sistema isolado, uma vez que permite destacar quantas unidades são atendidas onde a microgrid está instalada.

\section{As usinas térmicas em Rondônia}

Nos primeiros anos de fornecimento de energia elétrica no Brasil, a rede de geração e distribuição era baseada em grupos geradores a diesel. Em 1981, no recém-criado estado de Rondônia, em plena Amazônia, as Centrais Elétricas do Norte do Brasil (Eletronorte) assumem o fornecimento de energia da cidade de Porto Velho, capital do estado. Em janeiro de 1987, foi inaugurada a primeira Pequena Central Hidrelétrica 
no sul de Rondônia. Desde a década de 1960, o fornecimento se manteve apoiado em usinas térmicas. Em julho de 1989, a primeira unidade da Usina Hidrelétrica Samuel, no Rio Jamari, entrou em operação. Ao longo dos anos 1990, no interior do estado, existiam cerca de 72 usinas térmicas que operavam em áreas isoladas (figura 1). Atualmente, existem 25 sistemas isolados de geração de energia, minirredes (microgrids) sem conexão ao Sistema Interligado Nacional (SIN), que atendem cerca de 40 localidades isoladas [2].

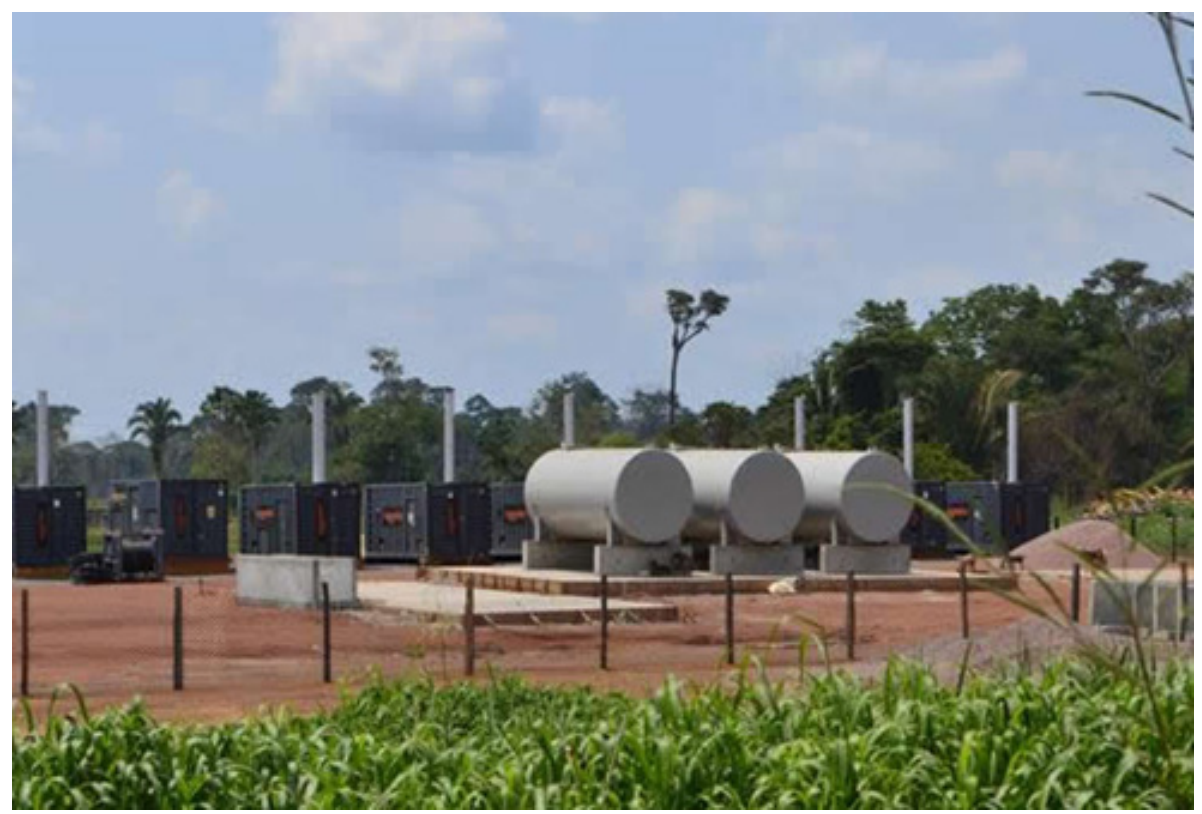

Figura 1. Usina Térmica de Vista Alegre do Abunã Fonte: [3]

De acordo com a Agência Nacional de Energia Elétrica (Aneel), as três grandes usinas do estado de Rondônia (Jirau, Samuel e Santo Antônio) têm capacidade de geração outorgada de $6,7 \mathrm{GW}$, e grande parte da energia gerada é entregue ao SIN para atender os mercados na região Sul e Sudeste do Brasil. O sistema isolado abastecido pelas usinas térmicas atende o segmento residencial e rural dos consumidores [4] (tabela 1).

Tabela 1. Concessionárias do sistema isolado em Rondônia

\begin{tabular}{|c|c|c|c|}
\hline Sistema isolado & Município & Concessionária & Unidades consumidoras \\
\hline Alvorada D'Oeste & Alvorada D’Oeste & Brasil Bio Fuels & 5.064 \\
\hline Buritis & Buritis & Grupo Rovema & 10.272 \\
\hline
\end{tabular}




\begin{tabular}{|c|c|c|c|}
\hline Sistema isolado & Município & Concessionária & Unidades consumidoras \\
\hline Calama & Porto Velho & Brasil Bio Fuels & 350 \\
\hline Campo Novo de Rondônia & Campo Novo de Rondônia & Grupo Rovema & 2.287 \\
\hline Conceição da Galera & Porto Velho & Brasil Bio Fuels & 28 \\
\hline Costa Marques & Costa Marques & Grupo Rovema & 2.373 \\
\hline Cujubim & Cujubim & Grupo Rovema & 4.320 \\
\hline Demarcação & Porto Velho & Brasil Bio Fuels & 37 \\
\hline Izidolândia & Alta Floresta D'Oeste & Grupo Rovema & 435 \\
\hline Machadinho & Machadinho & Grupo Rovema & 8.924 \\
\hline Maici & Porto Velho & Brasil Bio Fuels & 15 \\
\hline Nazaré & Porto Velho & Brasil Bio Fuels & 105 \\
\hline Nova Califórnia & Porto Velho & Grupo Rovema & 1.233 \\
\hline Pacarana & Espigão do Oeste & Grupo Rovema & 559 \\
\hline Pedras Negras & São Francisco do Guaporé & Brasil Bio Fuels & 5.064 \\
\hline Rolim de Moura do Guaporé & Alta Floresta D'Oeste & Brasil Bio Fuels & 128 \\
\hline Santa Catarina & Porto Velho & Brasil Bio Fuels & 43 \\
\hline São Carlos & Porto Velho & Grupo Rovema & 302 \\
\hline São Francisco do Guaporé & São Francisco do Guaporé & Grupo Rovema & 4.582 \\
\hline Surpresa & Guajará-Mirim & Brasil Bio Fuels & 240 \\
\hline União Bandeirantes & Porto Velho & Grupo Rovema & 1.287 \\
\hline Urucumacuã & Pimenta Bueno & Grupo Rovema & 84 \\
\hline Vale do Anari & Vale do Anari & Grupo Rovema & 3.054 \\
\hline Vila Extrema & Porto Velho & Grupo Rovema & 2.123 \\
\hline Vista Alegre do Abunã & Porto Velho & Grupo Rovema & 1.104 \\
\hline
\end{tabular}

Fonte: [4]

A eletricidade anual consumida por todo o sistema isolado de Rondônia alcança o montante de cerca de $335,6 \mathrm{GWh}$, o que representa apenas $6,2 \%$ do total da energia bruta requerida. Por sua vez, a usina hidrelétrica de Santo Antônio, em Porto Velho-RO, tem potência instalada de $3.568 \mathrm{MW}$ e pode gerar até $31,2 \mathrm{TWh}$, com funcionamento 24 horas por dia, em 365 dias do ano. A energia anual consumida pelo sistema isolado de Rondônia representa pouco mais de 1,07 \% de toda a energia anual gerada em Santo Antônio (tabela 2). 
210 Cristiano Torres do Amaral, Artur de Souza Moret y José Manuel Carvalho Marta

Tabela 2. Localidades com sistemas isolados em Rondônia

\begin{tabular}{|c|c|c|c|c|c|c|}
\hline \multirow[t]{2}{*}{ Sistema } & \multicolumn{2}{|c|}{ Energia anual } & \multirow{2}{*}{$\begin{array}{c}\text { Demanda } \\
\text { máxima anual } \\
M W\end{array}$} & \multirow{2}{*}{$\begin{array}{c}\text { Limite de consu- } \\
\text { mo especifico } \\
\text { m3/MWh }\end{array}$} & \multirow{2}{*}{$\begin{array}{c}\text { GT PIE } \\
M W h\end{array}$} & \multirow{2}{*}{$\begin{array}{c}\text { Cobertura } \\
\text { CCC } \\
m 3\end{array}$} \\
\hline & $M W h$ & MW médio & & & & \\
\hline Alvorada D'Oeste & 20.688 & 2 & 3,616 & 0,283 & & \\
\hline Buritis & 78.289 & 9 & 13,957 & 0,253 & & \\
\hline Campo Novo de Rondônia & 9.181 & 1 & 1,602 & 0,283 & & \\
\hline Conceição da Galeia & 90 & & 0,031 & 0,404 & 21 & 9 \\
\hline Costa Marques & 20.887 & 2 & 3,705 & 0,283 & & \\
\hline Cujubim & 32.892 & 4 & 8,188 & 0,253 & & \\
\hline Demarcação & 301 & & 0,120 & 0,349 & 66 & 23 \\
\hline Izidolândia & 1.281 & & 0,281 & 0,296 & & \\
\hline Machadinho & 52.099 & 6 & 9,316 & 0,253 & & \\
\hline Maici & 35 & & 0,011 & 0,404 & 8 & 3 \\
\hline Nazaré & 948 & & 0,331 & 0,329 & 223 & 73 \\
\hline Nova Califórnia & 8.912 & 1 & 2,676 & 0,283 & & \\
\hline Pacarana & 3.618 & & 1,195 & 0,289 & 32 & 11 \\
\hline Pedras Negras & 176 & & 0,050 & 0,349 & 192 & 63 \\
\hline $\begin{array}{l}\text { Rolim de Moura do } \\
\text { Guaponé }\end{array}$ & 926 & & 0,190 & 0,329 & 192 & 63 \\
\hline Santa Catarina & 282 & & 0,062 & 0,349 & 442 & 128 \\
\hline São Carlos & 2.080 & & 0,468 & 0,289 & & \\
\hline São Francisco & 35.739 & 4 & 7,075 & 0,283 & 240 & 79 \\
\hline Surpresa & 1.045 & & 0,221 & 0,329 & & \\
\hline Uniao Bandeirantes & 13.634 & 2 & 3,119 & 0,283 & & \\
\hline Urucumacuã & 1.837 & & 0,396 & 0,296 & & \\
\hline Vale do Anari & 13.337 & 2 & 2,346 & 0,283 & & \\
\hline Vila Extrema & 13.506 & 2 & 2,363 & 0,283 & & \\
\hline Vista Alegre do Abunã & 20.933 & 2 & 5,687 & 0,283 & & \\
\hline
\end{tabular}

\section{A gestão das usinas térmicas em Rondônia}

o sistema isolado em Rondônia é formado por microgrids que possuem a geração de energia elétrica a diesel. Esse sistema é operado por duas empresas concessionárias: 
Rovema Energia e Brasil Bio Fuels (BBF). A Rovema Energia administra 15 unidades, e a BBF é responsável por 10 usinas do sistema isolado (figura 2).

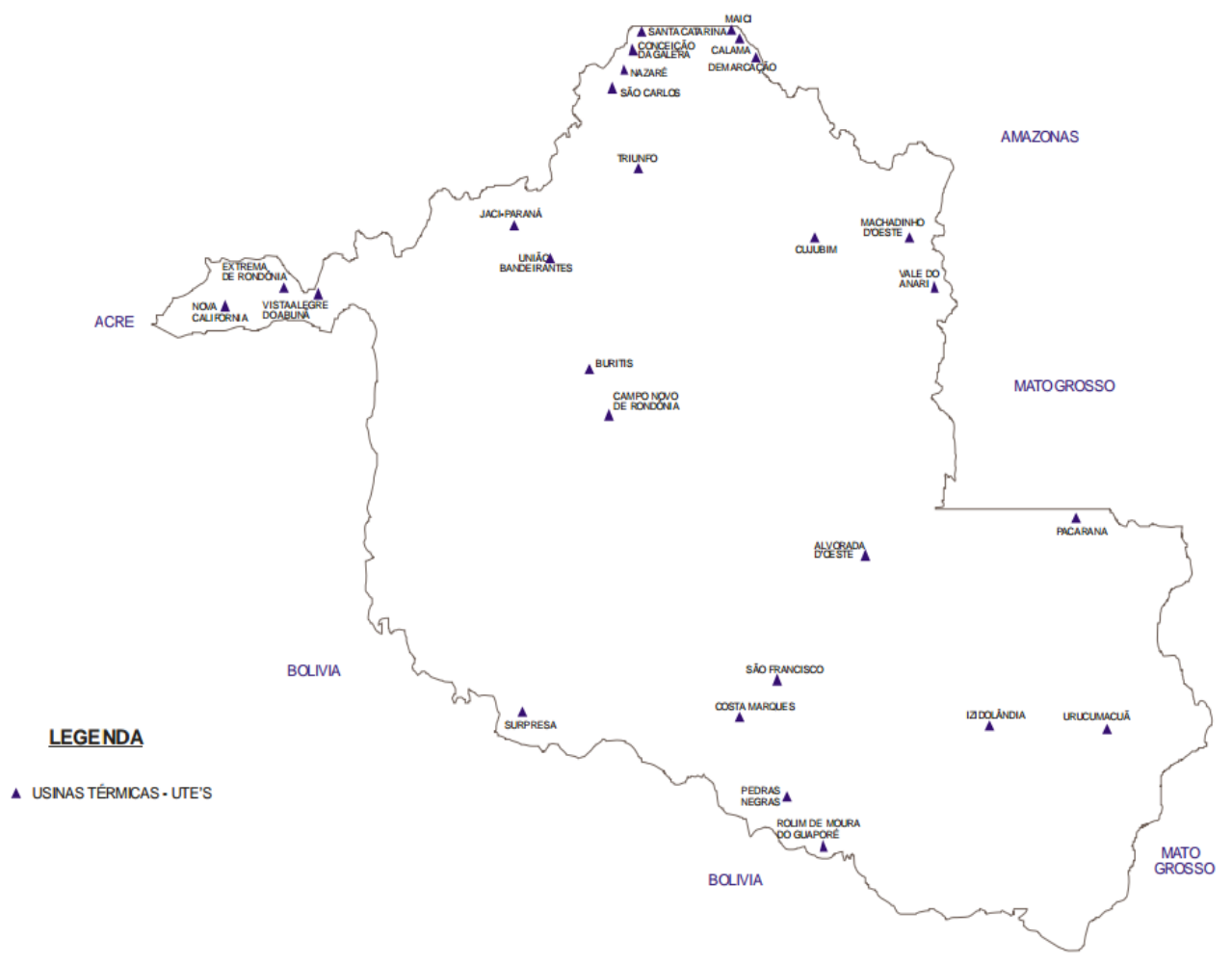

Figura 2. Usinas térmicas em Rondônia

Fonte: [4]

De acordo com o Relatório do Plano Anual de Operações dos Sistemas Isolados da Ceron-Eletrobras, gestora do sistema isolado, existe a previsão de interligação gradual do sistema isolado. As obras com recursos subsidiados pela Conta Consumo de Combustível devem atender as localidades com maior número de habitantes (figura 3).

As usinas térmicas foram instaladas com grupos geradores dispostos dentro de contêineres. As equipes de manutenção estão sediadas em Porto Velho (Rovema) e Ji-Paraná (BBF). De acordo com as empresas, a estrutura metálica é revestida com espuma termoacústica, que é capaz de atenuar os ruídos do motor do equipamento. Os motores estão instalados em uma estrutura com revestimento acústico em contêineres, mas produzem ruídos de cerca de 55 a 75 dB quando estão na sua carga máxima, conforme informado pelos responsáveis das equipes de manutenção das concessionárias [5]. 


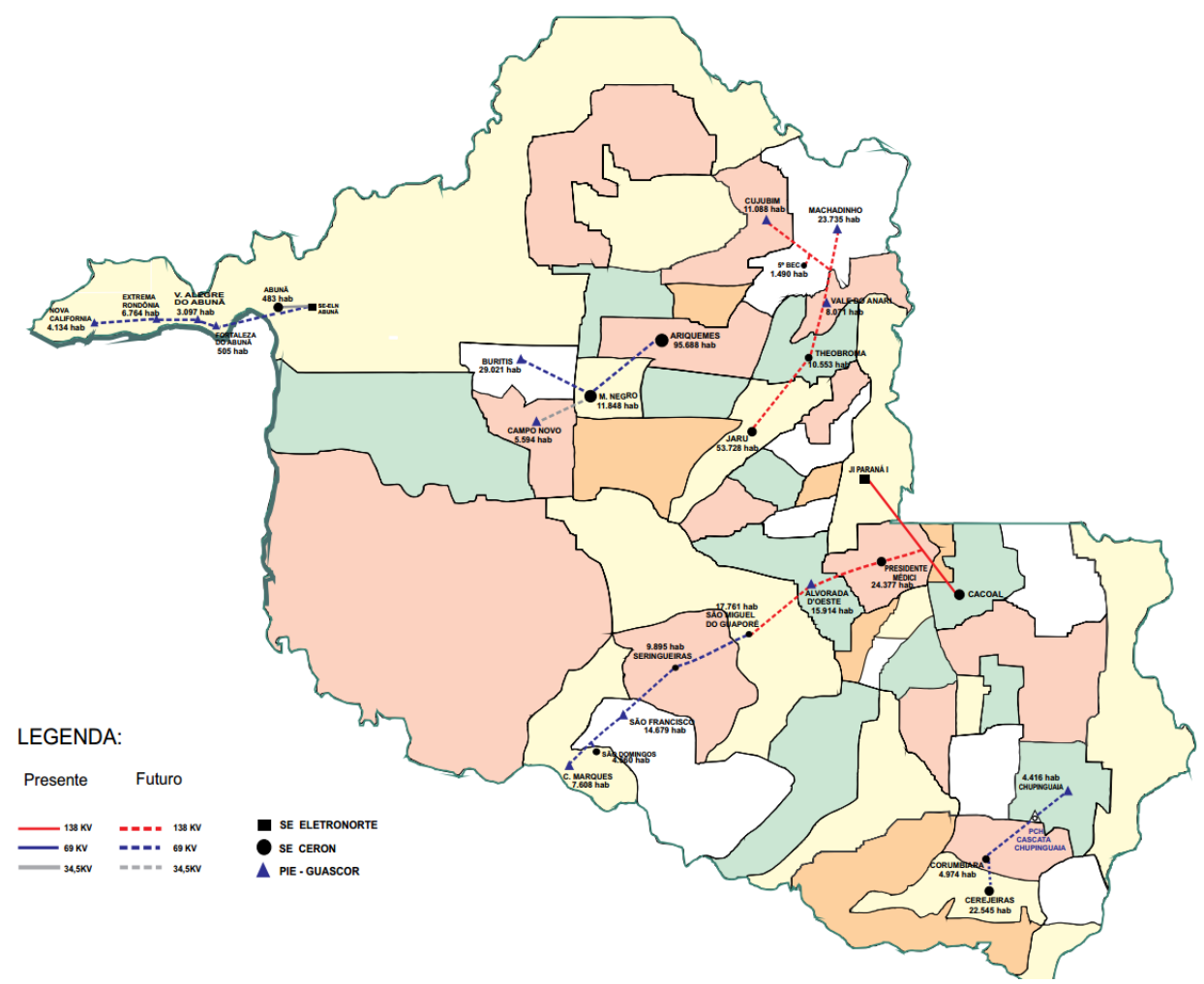

Figura 3. Planejamento de interligação do sistema isolado

Fonte: [2]

A água utilizada nos sistemas isolados é fornecida pela concessionária local e, quando a rede não está disponível, são utilizados poços amazônicos. A água descartada é enviada para as fossas sépticas. $O$ tanque de combustível e a bacia de contenção têm capacidade para até $110 \%$ de todo o volume de fluidos do gerador, incluindo o diesel [5].

A equipe de manutenção é formada por cerca de 15 pessoas contratadas diretamente em cada uma das empresas para atender todas as microgrids. Nessas unidades, é produzida cerca de uma tonelada de lixo sólido e líquido, que é recolhida para o descarte especializado. Os sistemas são avaliados trimestralmente para o monitoramento do impacto, a manutenção preventiva e, quando necessário, a manutenção corretiva [6].

\section{MATERIAIS E MÉTODOS}

A área da pesquisa está delimitada pelo estado de Rondônia, onde estão localizados os sistemas isolados de energia, ou seja, as microgrids. O estado de Rondônia é formado por 52 municípios e, nessas localidades, existem 25 sistemas isolados em operação. 
Os dados das unidades gestoras foram obtidos por meio de levantamento eletrônico submetido aos responsáveis técnicos dos sistemas microgrids e de consulta a documentos oficiais. Os dados apresentam itens quantitativos acerca dos sistemas isolados instalados em Rondônia, apurando de maneira censitária os dados das variáveis ambientais desta pesquisa $[7,8]$.

Um índice de sustentabilidade em sistemas microgrids precisa descrever diferentes dimensões, as quais contemplam aspectos sociais, econômicos e ambientais, auxiliam na gestão e orientam investimentos [8,9]. Além disso, o cálculo deve ser simples e acessível para todos. Um índice de sustentabilidade é formado por um conjunto de indicadores, obtidos de variáveis que descrevem elementos de comparação dos níveis de sustentabilidade dos sistemas microgrids.

Nesta pesquisa, os indicadores são balizados pelos temas de sustentabilidade que abordam a relação ambiental, econômica, social de um sistema microgrid e são considerados 25 itens. As variáveis foram agrupadas por tema, e o conjunto de dados foi classificado em três níveis. Essa metodologia adota uma adaptação da escala Likert com resposta psicométrica, com a avaliação em três níveis $(0,0 ; 0,5$ e 1,0). A variável com maior sustentabilidade, de acordo com as referências e recomendações internacionais, recebeu nota 1,0 - valor máximo. O nível intermediário, que até a metade do valor de referência, recebeu nota 0,5 e, acima deste, a menor nota 0,0 - valor mínimo. A maior nota foi atribuída conforme a contribuição da variável no desenvolvimento local [8].

Por exemplo, é desejável que a taxa de mortalidade infantil seja nula ou próxima de zero, logo a localidade com os índices até o limite (10/1.000 nascidos - com observado em regiões mais desenvolvidas) recebeu nota 1,0 . Para índices até $50 \%$ da referência, foi atribuído valor 0,5 . Acima desse valor, foi atribuído valor 0,0 . Por sua vez, os investimentos em educação ambiental devem ser maximizados e, nesse caso, quanto maior o valor investido por ligação de energia, mais próximo de 1,0 será o valor da variável [9].

Após a classificação em três níveis $(0 ; 0,5 ; 1,0)$, foi calculada a média das variáveis e, a partir dessa média, foram obtidos os indicadores de sustentabilidade para cada tema (ambiental, econômico e social). O Índice Geral de Sustentabilidade (IGS) foi elaborado com base na média do somatório dos indicadores de sustentabilidade (ambiental — IA, econômico - IE e social - IS). Cada indicador apresenta contribuição de 33 \% no resultado final do IGS.

Para IGS maior ou igual a 0,8 , será considerada a alta sustentabilidade do sistema microgrid. Para IGS entre 0,8 e 0,6 , a sustentabilidade será considerada média e, para IGS menor que 0,6 , o sistema microgrid receberá classificação de baixa sustentabilidade. IGS abaixo de 0,4 será considerado crítico (tabela 3) $[9,10]$. 
Tabela 3. Níveis de sustentabilidade dos sistemas microgrids

\begin{tabular}{lc}
\hline \multicolumn{1}{c}{ IGS } & Classificação \\
\hline IGS $>0,8$ & Alta \\
\hline $0,8 \geq$ IGS $>0,6$ & Média \\
\hline $0,6 \geq$ IGS $>0,4$ & Baixa \\
\hline IGS $\leq 0,4$ & Crítica \\
\hline \multicolumn{2}{c}{ Fonte: $[9,10]$}
\end{tabular}

Nesta pesquisa, foram utilizados dados que descrevem as variáveis dos sistemas microgrids no estado de Rondônia a partir de 2010 (Censo do Instituto Brasileiro de Geografia e Estatística - IBGE) até 2018. Para cada variável, existe um objetivo específico, que pretende mensurar o impacto de sustentabilidade dos sistemas microgrids.

Ao final, será simulado o impacto da substituição do combustível fóssil por energia fotovoltaica nos indicadores de sustentabilidade na geração de energia das áreas isoladas (tabelas 4, 5 e 6).

Tabela 4. Composição das variáveis ambientais

\begin{tabular}{|c|c|c|c|c|c|}
\hline Sigla Variáveis & Descrição & Objetivo & Unidade & Referência & Fonte \\
\hline IA1 Emissão de $\mathrm{CO}_{2}$ & $\begin{array}{l}\text { Estimativa de emissão de } \mathrm{CO}_{2} \\
\text { que considera o consumo de } \\
\text { diesel. }\end{array}$ & $\begin{array}{l}\text { Avaliar a contribuição do empreen- } \\
\text { dimento na emissão de GEE (CO2). }\end{array}$ & $\begin{array}{c}\text { Tonelada/ } \\
\text { kWh }\end{array}$ & $4 \mathrm{~g} / \mathrm{kWh}$ & {$[11,12,13]$} \\
\hline IA2 Emissão de $\mathrm{CH}_{4}$ & $\begin{array}{l}\text { Estimativa de emissão de } \mathrm{CH} 4 \\
\text { que considera o consumo de } \\
\text { diesel. }\end{array}$ & $\begin{array}{l}\text { Avaliar a contribuição do empreen- } \\
\text { dimento na emissão de GEE (CH4). }\end{array}$ & $\begin{array}{c}\text { Tonelada/ } \\
\text { kWh }\end{array}$ & $\begin{array}{l}0,46 \mathrm{~g} / \\
\mathrm{kWh}\end{array}$ & {$[11,12]$} \\
\hline IA3 Emissão de $\mathrm{NO}_{x}$ & $\begin{array}{l}\text { Estimativa de emissão de } \mathrm{NO}_{\mathrm{x}} \\
\text { que considera o consumo de } \\
\text { diesel. }\end{array}$ & $\begin{array}{l}\text { Avaliar a contribuição do empreen- } \\
\text { dimento na emissão de GEE }\left(\mathrm{NO}_{x}\right) \text {. }\end{array}$ & $\begin{array}{c}\text { Tonelada/ } \\
\text { kWh }\end{array}$ & $\begin{array}{l}1,8 \mathrm{~g} / \\
\mathrm{kWh}\end{array}$ & {$[11,12]$} \\
\hline IA4 Emissão de $\mathrm{SO}_{2}$ & $\begin{array}{l}\text { Estimativa de emissão de } \mathrm{SO}_{2} \\
\text { que considera o consumo de } \\
\text { diesel. }\end{array}$ & $\begin{array}{l}\text { Avaliar a contribuição do empreen- } \\
\text { dimento na emissão de GEE }\left(\mathrm{SO}_{2}\right) \text {. }\end{array}$ & $\begin{array}{c}\text { Tonelada/ } \\
\mathrm{kWh}\end{array}$ & $\begin{array}{l}1,0 \mathrm{~g} / \\
\mathrm{kWh}\end{array}$ & {$[11,12]$} \\
\hline IA5 $\begin{array}{l}\text { Investimento } \\
\text { socioambiental }\end{array}$ & $\begin{array}{l}\text { Investimento em ações socioam- } \\
\text { bientais. }\end{array}$ & $\begin{array}{l}\text { Avaliar o investimento da con- } \\
\text { cessionária para ações socioam- } \\
\text { bientais. }\end{array}$ & $\begin{array}{l}\text { Reais/ } \\
\text { Ligação }\end{array}$ & $\begin{array}{c}\mathrm{R} \$ \\
\text { 300,00/ } \\
\text { Ligação }\end{array}$ & {$[11,14]$} \\
\hline IA66 $\begin{array}{l}\text { Impacto de } \\
\text { recuperação }\end{array}$ & $\begin{array}{l}\text { Impacto das ações do plano de } \\
\text { recuperação. }\end{array}$ & $\begin{array}{l}\text { Avaliar a aplicação do plano de re- } \\
\text { cuperação do empreendimento. }\end{array}$ & Unidade & $\mathrm{CF}$ & {$[6,15]$} \\
\hline IA7 Plano de ação & Impacto do plano de ação. & $\begin{array}{l}\text { Avaliar a execução do plano de } \\
\text { ação do empreendimento. }\end{array}$ & Unidade & $\mathrm{CF}$ & {$[6,15]$} \\
\hline $\begin{array}{l}\text { IA8 } 8 \text { Mitigação de } \\
\text { riscos }\end{array}$ & $\begin{array}{l}\text { Impacto das ações do plano de } \\
\text { mitigação de riscos. }\end{array}$ & $\begin{array}{l}\text { Avaliar a execução do plano de } \\
\text { mitigação de riscos do empreen- } \\
\text { dimento. }\end{array}$ & Unidade & $\mathrm{CF}$ & {$[6,15]$} \\
\hline IA9 Resiliência & $\begin{array}{l}\text { Impacto das ações do plano de } \\
\text { resiliência. }\end{array}$ & $\begin{array}{l}\text { Avaliar a execução do plano de re- } \\
\text { siliência do empreendimento. }\end{array}$ & Unidade & $\mathrm{CF}$ & {$[6,5]$} \\
\hline
\end{tabular}




\begin{tabular}{|c|c|c|c|c|c|c|}
\hline Sigla & Variáveis & Descrição & Objetivo & Unidade & Referência & Fonte \\
\hline IA10 & $\begin{array}{l}\text { mpacto de } \\
\text { nonitoramento }\end{array}$ & $\begin{array}{l}\text { Impacto das atividades de mo- } \\
\text { nitoramento do plano de ação. }\end{array}$ & $\begin{array}{l}\text { Avaliar o monitoramento do pla- } \\
\text { no de ação do empreendimento. }\end{array}$ & Unidade & $\mathrm{CF}$ & {$[6,15]$} \\
\hline
\end{tabular}

CF — Constituição Federal do Brasil (art. 225, $§ 1^{\circ}$, inciso IV).

Fonte: elaboração própria.

Tabela 5. Composição das variáveis sociais

\begin{tabular}{|c|c|c|c|c|c|c|}
\hline Sigla & Variáveis & Descrição & Objetivo & Unidade & Referência & Fonte \\
\hline IS1 & $\begin{array}{l}\text { Estabelecimen- } \\
\text { tos de saúde do } \\
\text { SUS }\end{array}$ & $\begin{array}{l}\text { Unidades de atendimento pú- } \\
\text { blico no município por número } \\
\text { de ligações. }\end{array}$ & $\begin{array}{l}\text { Avaliar a abrangência do empreen- } \\
\text { dimento para atender unidades de } \\
\text { saúde pública. }\end{array}$ & $\begin{array}{l}\text { Unidade/ } \\
\text { Ligações }\end{array}$ & $\begin{array}{l}1 / 30.000 \\
\text { habitantes }\end{array}$ & {$[16$,} \\
\hline IS2 & $\begin{array}{l}\text { Índice de ma- } \\
\text { lária }\end{array}$ & $\begin{array}{l}\text { Percentual de exames positivos } \\
\text { de malária realizados no muni- } \\
\text { cípio por número de ligações. }\end{array}$ & $\begin{array}{l}\text { Avaliar o índice de malária } \\
\text { na área de abrangência do } \\
\text { empreendimento. }\end{array}$ & $\begin{array}{l}\text { Percen- } \\
\text { tagem/ } \\
\text { Ligações }\end{array}$ & $\begin{array}{c}<90 \% \\
\text { de } 6.734 \\
\text { casos de } \\
\text { Rondônia }\end{array}$ & {$[16$, } \\
\hline IS3 & $\begin{array}{l}\text { Mortalidade } \\
\text { infantil }\end{array}$ & $\begin{array}{l}\text { Percentual de mortalidade in- } \\
\text { fantil em relação aos nascidos } \\
\text { vivos no município por número } \\
\text { de ligações. }\end{array}$ & $\begin{array}{l}\text { Avaliar a taxa de mortalidade } \\
\text { infantil na área de abrangência } \\
\text { do empreendimento. }\end{array}$ & $\begin{array}{l}\text { Percen- } \\
\text { tagem/ } \\
\text { Ligações }\end{array}$ & $\begin{array}{l}10 / 1.000 \\
\text { nascidos }\end{array}$ & {$[16,17,18]$} \\
\hline IS4 & $\begin{array}{l}\text { Estabelecimen- } \\
\text { tos de ensino } \\
\text { fundamental }\end{array}$ & $\begin{array}{l}\text { Unidades de ensino fundamen- } \\
\text { tal no município por número } \\
\text { de ligações. }\end{array}$ & $\begin{array}{l}\text { Avaliar a abrangência do empreen- } \\
\text { dimento para atender unidades de } \\
\text { ensino público primário. }\end{array}$ & $\begin{array}{l}\text { Unidade/ } \\
\text { Ligações }\end{array}$ & $100 \%$ & {$[19,20]$} \\
\hline IS5 & $\begin{array}{l}\text { Estudantes do } \\
\text { ensino funda- } \\
\text { mental }\end{array}$ & $\begin{array}{l}\text { Estudantes no ensino funda- } \\
\text { mental no município por núme- } \\
\text { ro de ligações. }\end{array}$ & $\begin{array}{l}\text { Avaliar a abrangência do empreen- } \\
\text { dimento para a ampliação da edu- } \\
\text { cação primária. }\end{array}$ & $\begin{array}{l}\text { Unidade/ } \\
\text { Ligações }\end{array}$ & $95 \%$ & {$[20]$} \\
\hline IS6 & $\begin{array}{l}\text { Estudantes do } \\
\text { ensino médio }\end{array}$ & $\begin{array}{l}\text { Estudantes no ensino mé- } \\
\text { dio no município por número } \\
\text { de ligações. }\end{array}$ & $\begin{array}{l}\text { Avaliar a abrangência do empreen- } \\
\text { dimento para a ampliação do ensi- } \\
\text { no médio. }\end{array}$ & $\begin{array}{l}\text { Unidade/ } \\
\text { Ligações }\end{array}$ & $85 \%$ & {$[20]$} \\
\hline IS7 & $\begin{array}{l}\text { Adultos sem } \\
\text { instrução }\end{array}$ & $\begin{array}{l}\text { Adultos que não concluíram o } \\
\text { ensino fundamental no muni- } \\
\text { cípio por número de ligações. }\end{array}$ & $\begin{array}{l}\text { Avaliar a abrangência do empreen- } \\
\text { dimento para a redução do anal- } \\
\text { fabetismo. }\end{array}$ & $\begin{array}{l}\text { Unidade/ } \\
\text { Ligações }\end{array}$ & $93,5 \%$ & [20] \\
\hline IS8 & $\begin{array}{l}\text { P o p u } 1 \text { a ção } \\
\text { indígena do } \\
\text { município }\end{array}$ & $\begin{array}{l}\text { População indígena do municí- } \\
\text { pio por número de ligações. }\end{array}$ & $\begin{array}{l}\text { Avaliar a abrangência do empreen- } \\
\text { dimento para contemplar a popula- } \\
\text { ção indígena. }\end{array}$ & $\begin{array}{l}\text { Unidade/ } \\
\text { Ligações }\end{array}$ & $100 \%$ & {$[16]$} \\
\hline
\end{tabular}

SUS - Sistema Único de Saúde do Brasil.

Fonte: elaboração própria.

Tabela 6. Composição das variáveis econômicas

\begin{tabular}{|c|c|c|c|c|c|c|}
\hline Sigla & Variáveis & Descrição & Objetivo & Unidade & Referência & a Fonte \\
\hline IE1 & Investimento & $\begin{array}{l}\text { Investimento necessário para a im- } \\
\text { plantação da microgrid. }\end{array}$ & $\begin{array}{l}\text { Avaliar a amplitude financei- } \\
\text { ra do investimento. }\end{array}$ & Reais & $\begin{array}{c}\mathrm{R} \$ \\
10.000,00\end{array}$ & {$[11,14,21]$} \\
\hline IE2 & $\begin{array}{l}\text { Empregos } \mathrm{f} \\
\text { mais }\end{array}$ & $\begin{array}{l}\text { for- Empregos no município onde exis- } \\
\text { te a microgrid. }\end{array}$ & $\begin{array}{l}\text { Avaliar o impacto do em- } \\
\text { preendimento para a geração } \\
\text { de empregos. }\end{array}$ & $\begin{array}{l}\text { Unidade/ } \\
\text { Ligação }\end{array}$ & 500 & {$[11,14,21]$} \\
\hline
\end{tabular}




\begin{tabular}{|c|c|c|c|c|c|c|}
\hline Sigla & Variáveis & Descrição & Objetivo & Unidade & Referência & Fonte \\
\hline IE3 & Renda per capta & $\begin{array}{l}\text { Renda per capta no município aten- } \\
\text { dido pela microgrid. }\end{array}$ & $\begin{array}{l}\text { Avaliar a renda local onde foi } \\
\text { feito o investimento. }\end{array}$ & $\begin{array}{l}\text { Reais/ } \\
\text { Habitante/ } \\
\text { Ligação }\end{array}$ & $\mathrm{R} \$ 50,00$ & [19] \\
\hline IE4 & $\begin{array}{l}\text { Ligações de ele- } \\
\text { tricidade }\end{array}$ & $\begin{array}{l}\text { Ligações de eletricidade } \\
\text { em residências. }\end{array}$ & $\begin{array}{l}\text { Avaliar a contribuição do em- } \\
\text { preendimento para a univer- } \\
\text { salização do acesso à eletri- } \\
\text { cidade. }\end{array}$ & $\begin{array}{l}\text { Unidade/ } \\
\text { Hab }\end{array}$ & $25 \%$ & {$[11,15]$} \\
\hline IE5 & $\begin{array}{l}\text { Custo da tecno- } \\
\text { logia }\end{array}$ & $\begin{array}{l}\text { Custo da tecnologia para a implanta- } \\
\text { ção da microgrid. }\end{array}$ & $\begin{array}{l}\text { Avaliar o impacto do em- } \\
\text { preendimento no custo de } \\
\text { tecnologia. }\end{array}$ & Reais/kWh & $\begin{array}{l}\mathrm{R} \$ 34,00 / \\
\mathrm{MWh}\end{array}$ & {$[11,14,22]$} \\
\hline IE6 & $\begin{array}{l}\text { Custo da eletri- } \\
\text { cidade }\end{array}$ & $\begin{array}{l}\text { Custo da eletricidade fornecida } \\
\text { à população. }\end{array}$ & $\begin{array}{l}\text { Avaliar o custo do serviço } \\
\text { prestado na localidade. }\end{array}$ & Reais/kWh & $\begin{array}{l}\mathrm{R} \$ 34,00 / \\
\mathrm{MWh}\end{array}$ & {$[11,14,22]$} \\
\hline IE7 & Custo de impacto & $\begin{array}{l}\text { Relação direta entre o investimen- } \\
\text { to e o número de ligações atendidas. }\end{array}$ & $\begin{array}{l}\text { Avaliar a dimensão do inves- } \\
\text { timento em relação à eletrifi- } \\
\text { cação do município. }\end{array}$ & Reais/kWh & $\begin{array}{l}\mathrm{R} \$ 34,00 / \\
\mathrm{MWh}\end{array}$ & {$[11,14]$} \\
\hline
\end{tabular}

Fonte: elaboração própria.

\section{APRESENTAÇÃO DOS DADOS E ANÁLISE DOS RESULTADOS}

As variáveis IA1, IA2, IA3 e IA4 estão relacionadas com a emissão de GEE, e os dados foram obtidos no Relatório do Plano Anual de Operações dos Sistemas Isolados do Grupo Técnico Operacional da Região Norte. Esse documento oficial descreve o consumo anual de óleo diesel e a energia bruta gerada nos sistemas isolados [23]. Esses dados possibilitam estimar a emissão das variáveis conforme descrição detalhada na ISO 14.064 - Parte 2: Especificação e orientação a organizações para a quantificação e elaboração de relatórios de emissões e remoções de GEE.

No Brasil, a redução da emissão dos GEE está estabelecida no Decreto 7.390/2010, que institui a Política Nacional sobre Mudança do Clima e define o limite de 3.236 milhões ton $\mathrm{CO}_{2}$ eq, com projeção de 868 milhões de ton $\mathrm{CO}_{2}$ eq para o setor de energia. Por sua vez, os limites para a emissão de poluentes atmosféricos para as fontes fixas instaladas estão estabelecidos na Resolução 436/2011 do Conselho Nacional do Meio Ambiente. Neste trabalho, para essas variáveis, a sustentabilidade estará mais próxima do valor desejável $(1,0)$ quando a emissão alcançar valores estabelecidos na legislação.

A variável IA5 apresenta o valor de investimento em educação ambiental e, neste caso, conforme observado no Manual de Contabilidade do Setor Elétrico, da Aneel, não há um valor de referência. Contudo, é desejável o maior investimento em ações socioambientais em função do número de ligações, uma vez que descreverá a sustentabilidade da rede instalada [24].

De acordo com os responsáveis pela manutenção, os planos de ação, mitigação, recuperação e impacto dos sistemas isolados existem, entretanto eles não são públicos. 
Essas informações são estratégicas para a execução da concessão, uma vez que compõe a planilha de custos para prestação do serviço. Além disso, de acordo com os editais de licitação da Aneel publicados em seu portal, não há referências/exigências para tais projetos na execução dos serviços de geração de energia, apenas a obtenção das licenças ambientais [24].

A ausência de divulgação dos dados sobre o plano de recuperação de área (IA6), plano de ação (IA7), mitigação de riscos (IA8), resiliência (IA9) e impacto do monitoramento do empreendimento (IA10) resultaram na nota nula para essas variáveis. Essas informações também não estão detalhadas no demonstrativo anual da Ceron-Eletrobras [25].

Nenhum sistema isolado apresentou um indicador de sustentabilidade ambiental adequado. As microgrids não apresentaram indicadores de sustentabilidade ambiental, que ultrapassaram 0,4, e foram classificadas com sustentabilidade crítica. É um resultado insatisfatório para a gestão do sistema, uma vez que todo o parque instalado não possui sustentabilidade ambiental adequada para a manutenção do modelo de geração de energia com a utilização do diesel no estado de Rondônia.

A ausência de publicidade dos planos de ação, mitigação, recuperação e impacto também compromete a transparência da gestão ambiental dos sistemas isolados. É uma situação alarmante, uma vez que a sociedade civil não pode fiscalizar a execução dos contratos, bem como se prevenir de desastres ambientais que porventura possam ocorrer. As microgrids de Rolim de Moura do Guaporé, Urucumacuã, Nova Califórnia, São Carlos, Surpreza, Calama, Conceição da Galera, Demarcação, Maici, Nazaré e Santa Catarina apresentam indicadores de sustentabilidade social de nivel crítico (figura 4). O resultado observado nos indicadores de sustentabilidade social sugere a necessidade de expansão da planta de distribuição de eletricidade no estado. Quanto maior a capilaridade do sistema, maior será a contribuição da infraestrutura e a possibilidade de melhorar a qualidade de vida das pessoas nas comunidades.

As comunidades mais carentes de infraestrutura básica de eletricidade também apresentam as variáveis com maior vulnerabilidade social, isto é, maiores taxas de mortalidade infantil por número de ligações de energia; mais casos de malária por ligação de energia; entre outras.

Nas comunidades carentes, a ausência de energia elétrica dificulta ou restringe a instalação de unidades de saúde, escolas, hospitais etc. A população não tem acesso a remédios, vacinação, prevenção etc. As gestantes não têm acesso ao atendimento de pré-natal e ao parto acompanhado. Todos esses fatores contribuem para a deterioração das variáveis sociais que avaliam a contribuição social da microgrid. 
Os indicadores de sustentabilidade econômica apresentam resultados que descrevem a baixa sustentabilidade dos sistemas microgrids, visto que não contribuem para o desenvolvimento local. As variáveis que detalham o emprego formal, o custo de implantação e o investimento não apresentam resultados satisfatórios, e, ainda, o custo da energia continua elevado. Essa situação é considerada crítica nos sistemas isolados de Conceição da Galera, Demarcação, Maici e Santa Catarina, com o menor indicador de sustentabilidade econômica observado.

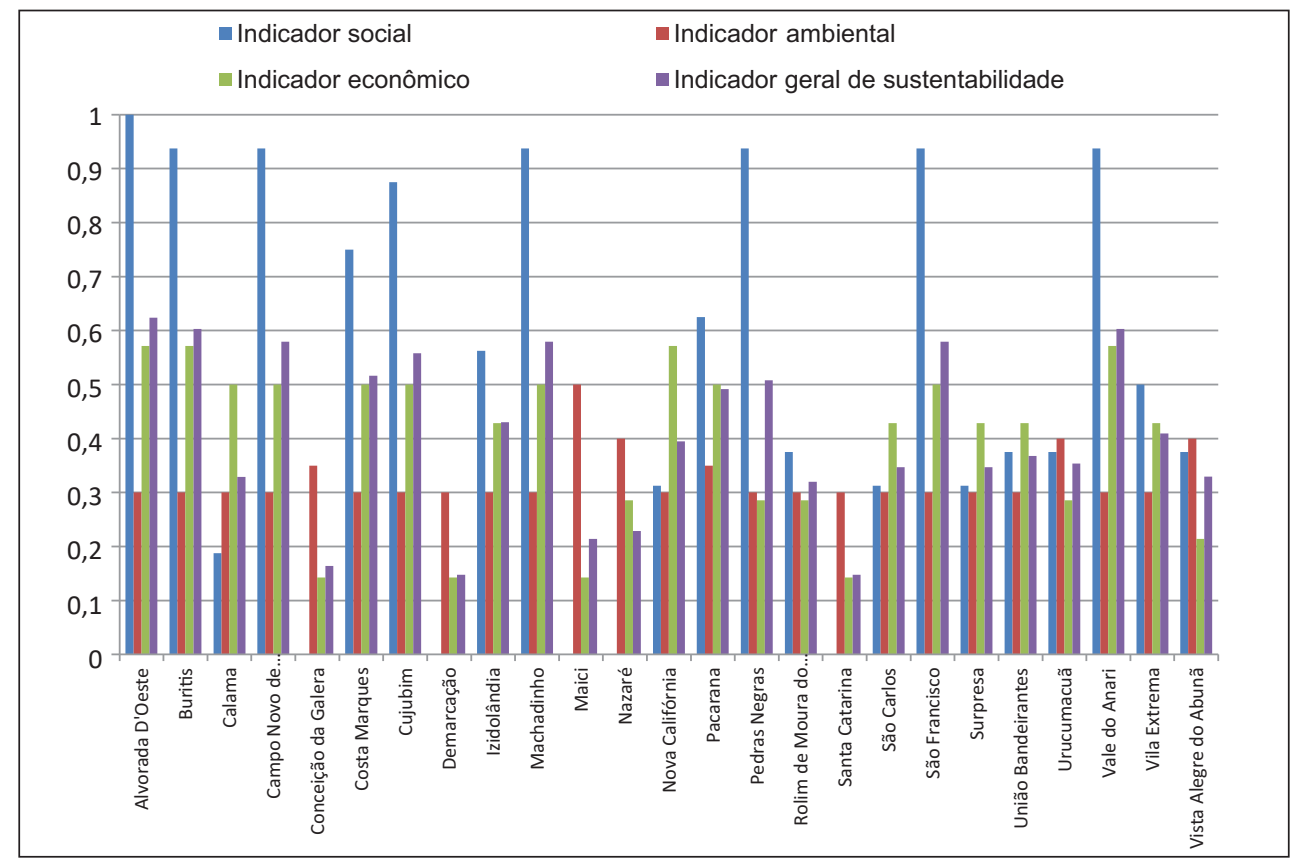

Figura 4. Indicador geral de sustentabilidade das microgrids

Fonte: elaboração própria.

\section{ANÁLISE DOS RESULTADOS}

Os indicadores utilizados neste modelo como ferramenta para medir a sustentabilidade dos sistemas microgrids nas áreas isoladas da Amazônia comprovam que o sistema de geração de energia elétrica baseado no combustível fóssil (diesel) em Rondônia é crítico, o que desperta a apreensão para o monitoramento e acompanhamento de sua operação. O IGS comprovou a baixa sustentabilidade dos sistemas instalados no estado nas dimensões ambiental, social e econômica.

A contribuição dos sistemas isolados para a operação sustentável local não é relevante, ao contrário, como demonstrado pelas variáveis ambientais, as microgrids de Rondônia contribuem para a emissão de GEE e, ainda, não possuem transparência 
na publicação de seus dados. Não há publicidade dos planos de ação, de mitigação, recuperação, resiliência e impacto. Essa é uma vulnerabilidade relevante. A comunidade deve conhecer e contribuir com a elaboração, acompanhamento e fiscalização desses documentos. O plano de ação é responsável por orientar os gestores na operação sustentável do empreendimento. O plano de mitigação apresenta estratégias para atenuar e corrigir prováveis efeitos colaterais decorrentes da operação do sistema isolado. E, caso ocorram sinistros, os planos de recuperação, resiliência e impacto devem prever ações para a correção rápida dos gestores, fiscais e autoridades. Portanto, é recomendável que tais documentos sejam públicos e de fácil acesso. Entretanto, conforme verificado nesta pesquisa, esses documentos não são disponibilizados pelas concessionárias e não há obrigação contratual para que isso ocorra.

É possível observar que, na maior parte das comunidades, os indicadores sociais não alcançam amplitude que possa garantir a manutenção/operação sustentável do sistema com a utilização do modelo atual. Isso significa dizer que os sistemas isolados de geração de energia elétrica alimentados com diesel não privilegiam e não contribuem para o desenvolvimento social local.

Os indicadores sociais não descrevem a cobertura e capilaridade das ligações de energia nas comunidades, essenciais para ampliar da qualidade de vida nas áreas isoladas. Os dados referentes aos estabelecimentos de saúde, ao índice de malária e à mortalidade infantil demonstram que a infraestrutura de energia elétrica não apresenta capilaridade suficiente para reduzir esses indicadores. Os baixos resultados dos indicadores associados à educação e ao trabalho nas comunidades isoladas também indicam que as metas de universalização do ensino não foram atingidas, principalmente onde ocorre pequena cobertura do sistema de distribuição de energia elétrica.

Os sistemas microgrids precisam adotar um modelo de geração sustentável nas áreas isoladas, integrando a comunidade e gerando renda. $\mathrm{O}$ modelo atual se resume a instalar motores geradores nas comunidades e levar combustível (diesel) até o ponto de operação. Quando ocorre uma pane, engenheiros e técnicos da área urbana de Rondônia são deslocados para os reparos. Nesse processo, os empregos gerados e a renda não alcançam a população local das áreas isoladas, por isso os indicadores econômicos também não alcançaram dimensões satisfatórias.

O uso da Conta Consumo de Combustível (CCC) para o custeio do diesel e a concessão da distribuição/operação para os conglomerados de empresas de energia favorece grupos econômicos que não têm vínculo com as comunidades. Os resultados dos indicadores do custo da tecnologia, do custo de impacto e do custo da eletricidade apresentam o elevado custo da geração de energia baseada no diesel. Também é possível constatar que o investimento por ligação de eletricidade na área 
isolada, em algumas comunidades com geração a diesel, é maior que o valor médio de referência $(\mathrm{R} \$ 10.000,00)$.

A ferramenta de análise de sustentabilidade comprovou que os sistemas microgrids de Rondônia, baseados na geração a diesel, não contribuem para o desenvolvimento sustentável no estado. O modelo atual privilegia o custeio do combustível fóssil subsidiado que favorece a emissão de GEE, fomenta grandes conglomerados econômicos fora das comunidades e não contribui para o desenvolvimento local.

\section{CONCLUSÕES}

Os resultados observados nos indicadores de sustentabilidade social demonstram a limitação da cobertura da planta de distribuição de eletricidade em Rondônia. Os indicadores possibilitaram a classificação dos sistemas isolados em Rondônia, com a identificação de prioridades para a substituição das usinas térmicas por fontes de energia com recursos renováveis. As microgrids que utilizam recursos renováveis podem mudar esse cenário e promover a universalização da energia elétrica.

Os resultados indicadores ambientais são preocupantes. O resultado insatisfatório reflete o modelo de gestão do sistema isolado adotado em Rondônia. A sustentabilidade ambiental é frágil e não tem sustentação para a operação de longo prazo. Não há publicidade dos planos de ação, de mitigação, de recuperação e de impacto, o que compromete a fiscalização, a auditoria e a contribuição da sociedade para se prevenir de desastres ambientais que porventura possam ocorrer. Na verdade, não há sequer garantia que esses planos existam.

As microgrids apresentam indicadores de sustentabilidade social e econômico crítico e isso significa dizer que a contribuição para melhorar a qualidade de vida nas comunidades não é suficiente para aquecer a economia. As comunidades isoladas são as mais carentes de infraestrutura do estado e apresentam maior vulnerabilidade social. Nessas áreas, são verificadas as variáveis com as maiores taxas de mortalidade infantil, mais casos de malária e ausência de cobertura de postos de saúde. Se não existe energia elétrica, haverá restrição para o atendimento médico e hospitalar. Além disso, a população não terá acesso à medicação, à vacinação, à prevenção, entre outros serviços.

Comparando os resultados dos indicadores ambiental, social e econômico, é possível constatar o nível crítico de sustentabilidade das microgrids em Rondônia. O equilíbrio homem-natureza é abalado pela instalação de motores a diesel nas áreas isoladas da floresta na Amazônia. O modelo de geração de energia não privilegia recursos locais e sustentáveis, não promove a geração de empregos e renda, bem como não é suficiente para promover a expansão da infraestrutura básica. Os 
geradores possuem limitação técnica de capacidade de operação, a expansão é restrita, depende de subsídio da CCC, do transporte do combustível por longas distâncias e exige altos investimentos.

\section{REFERÊNCIAS}

[1] A. S. Moret. Rondônia: 2000-2013. São Paulo: Fundação Perseu Abramo, 2014, 116p.

[2] Ceron-Eletrobras, "Relatório de Demonstrações Financeiras e Administração 2015" [On-line], acesso 3 de julho 2019; Disponível: http://www.diof.ro.gov.br/data/uploads/2016/04/ DOE15_04_2016.pdf, 2016.

[3] J. Donizete, "Rovema energia inicia obras para novos motores geradores" [On-line], acesso 3 de julho 2019; Disponível: http://maoamiga-anuncios.com.br/ rovema-energia-inicia-obras-para-novos-motores-geradores-na-ponta-do-abuna, 2017.

[4] Eletrobras, "Plano anual de operações dos sistemas isolados para 2016 - Grupo Técnico Operacional da Região Norte — GTON" [On-line], acesso 5 de julho 2019; Disponível http:// www2.aneel.gov.br/aplicacoes/audiencia/arquivo/2015/083/documento/plano_anual_de_operacao_2016.pdf, 2016.

[5] Agrekko, “Geradores a diesel - Contêiner" [On-line], acesso 5 de julho 2019; Disponível: http://www.aggreko.com.br/produtos-servicos/aluguel-geradores-energia/aluguel-geradores-a-diesel/, 2017.

[6] C. T. Amaral "Entrevista com a equipe de manutenção do Sistema Isolado em 6 de abril de 2017", Aplicação de ferramenta de análise de sustentabilidade de sistemas microgrids na Amazônia, [On-line], acesso 5 de julho 2019; Disponível: http://www.pgdra.unir.br/ uploads/85796698/arquivos/Cristiano_Amaral_Tese_2016_2018.pdf, 2018.

[7] M. S. A. Alvorado e A. S. Moret, "Relatório de Indicadores para Análise de Sustentabilidade de Microgrids. FULBRIGHT/NEXUS 2016” [On-line], acesso 5 de julho 2019; Disponível: https://www.cies.org/grantee/artur-moret, 2018.

[8] G. Liu et al. "Development of a general sustainability indicator for renewable energy systems: A review", Renewable and Sustainable Energy Reviews”, v. 31, n. 3, pp. 611-621, março, 2014. DOI: https://doi.org/10.1016/j.rser.2013.12.038

[9] A. Yadoo, A. e H. Cruickshank, "The role for low carbon electrification technologies in poverty reduction and climate change strategies: A focus on renewable energy mini-grids with case studies in nepal, peru and kenya”, Energy Policy, v. 42, pp. 591-602, março, 2012. DOI: https://doi.org/10.1016/j.enpol.2011.12.029

[10] F. A. Vale et al. "Análise comparativa de indicadores de sustentabilidade entre os estados da Amazônia Legal”, Sustentabilidade em Debate, v. 9, n.1, p. 214-231, abril, 2018. DOI: https:// doi.org/10.18472/SustDeb.v9n1.2018.25621

[11] Ceron, "Projeto de Referência para Atendimento dos Sistemas Isolados com Previsão de Interligação da Eletrobras Distribuição Rondônia” [On-line], acesso 3 de julho 2019; Disponível: http://www2.aneel.gov.br/area.cfm?idArea=17\&idPerfil=8, 2017 
[12] ABNT. Gases de efeito estufa - Parte 2: Especificação e orientação a organizações para a quantificação e elaboração de relatórios de emissões e remoções de gases de efeito estufa, NBR-ISO 14.064, 2015.

[13] Brasil, "Lei Federal 12.187 — Institui a Política Nacional sobre Mudança Climática" [On-line], acesso 3 de julho 2019; Disponível: www.planalto.gov.br/ccivil_03/_Ato2007-2010/2009/Lei/ L12187.htm, 2009.

[14] Brasil, "Manual de Contabilidade do Setor Elétrico" [On-line], acesso 8 de julho de 2019; Disponível: http://www.aneel.gov.br/arquivos/PDF/MCSE_-_Revisão.pdf, 2015.

[15] Brasil, "Indicadores de Universalização" [On-line], acesso 15 de julho de 2019; Disponível: http://www.aneel.gov.br/indicadores-da-distribuicao, 2019.

[16] Sepog-RO, "Rondônia em números" [On-line], acesso 6 agosto de 2019, Disponível: http:// www.odr.ro.gov.br/home/stateperfil, 2018.

[17] OMS, “Global Technical Strategy for Malaria 2016-2030” [On-line], acesso 6 agosto de 2019; Disponível: http://www.who.int/malaria/publications/atoz/9789241564991/en, 2015.

[18] SUS, “Caderno do Estado de Rondônia" [On-line], acesso 6 agosto de 2019; Disponível: http://tabnet.datasus.gov.br/tabdata/cadernos/ro.htm, 2019.

[19] IBGE, "Censo Demográfico 2010. Resultados da Amostra" [On-line], acesso 6 agosto de 2019; Disponível: https://cidades.ibge.gov.br/v4/brasil/ro/, 2010.

[20] Inep, "Indicadores Educacionais — Resultados da Amostra" [On-line], acesso 1 agosto de 2019; Disponível: http://portal.inep.gov.br/indicadores-educacionais, 2018.

[21] TCU, "Relatório de Auditoria TC 013.066/2012-9", [On-line], acesso 3 agosto de 2019; Disponível: https://pesquisa.apps.tcu.gov.br/\#/documento/acordao-completo/*/KEY:ACORDAO-COMPLETO-1335610/DTRELEVANCIA\%20desc/0/sinonimos\%3Dfalse, 2014.

[22] EPE/MME, "Plano Decenal de Expansão de Energia 2026" [On-line], acesso 5 agosto de 2019; Disponível: http://www.epe.gov.br/pt/publicacoes-dados-abertos/publicacoes/ Plano-Decenal-de-Expansao-de-Energia-2026, 2018.

[23] Eletrobras, "Mapa do Sistema Interligado Nacional com PDE 2024” [On-line], acesso 1 agosto de 2019; Disponível: http://www.epe.gov.br/pt/publicacoes-dados-abertos/publicacoes/ mapa-do-sistema-interligado-nacional, 2016.

[24] Eletrobras, “Documentos do Leilão 010/2015” [On-line], acesso 6 agosto de 2019; Disponível: http://www2.aneel.gov.br/aplicacoes/editais_geracao/documentos_editais.cfm?IdProgramaEdital=142, 2015.

[25] EPE/MME, "Nota Técnica 06/2013 - Contratação de energia para sistemas isolados" [On-line], acesso 10 de julho de 2019; Disponível: http://www2.aneel.gov.br/aplicacoes/ editais_geracao/documentos/06.pdf, 2013. 